\title{
Rupture of a liposomal vesicle
}

\author{
Marco A. Idiart and Yan Levin \\ Instituto de Física, UFRGS, Caixa Postal 15051, CEP 91501-970, Porto Alegre, RS, Brazil
}

(Received 20 October 2003; published 23 June 2004)

\begin{abstract}
We discuss pore dynamics in osmotically stressed vesicles. A set of equations which govern the liposomal size, internal solute concentration, and pore diameter is solved numerically. We find that dependent on the internal solute concentration and vesicle size, liposomes can stay pore free, nucleate a short-lived pore, or nucleate a long-lived pore. The phase diagram of pore stability is constructed, and the different scaling regimes are deduced analytically.
\end{abstract}

DOI: 10.1103/PhysRevE.69.061922

PACS number(s): 87.16.Dg

\section{INTRODUCTION}

Liposomal vesicle consists of a lipid bilayer separating the interior volume, containing an aqueous solution, from an exterior suspension. The vesicle membrane allows for a free exchange of water between the interior and exterior of the liposome, with the flux determined by the membrane composition. On the other hand, the lipidic membrane strongly inhibits the passage of large molecules, in particular if they contain ionized groups.

Liposomes are of great theoretical interest as the simplest model of a biological cell. They are also of great practical importance as vehicles for drug delivery. In the latter case liposomes are designed to contain a specific drug or a gene needed to fight the disease. The liposomal affinity for infected tissue can be increased by varying the membrane composition or including ligands which bind to specific receptors.

If a vesicle containing high internal solute concentration is placed inside a dilute solution, the osmotic influx of solvent into the interior of a vesicle can lead to its rupture. Whether the rupture occurs depends on the membrane elasticity and on the internal solute concentration of the liposome. Rupture of the liposomal membrane results in the formation of pores [1]. This releases the membrane stress, but comes at a price of exposing the hydrophobic membrane interior (lipidic tails) to water. Once a pore is formed, the internal content of the vesicle begins to leak out, resulting in a decrease of membrane tension and eventual pore closure. We find that depending on the vesicle size and internal concentration of solute, pores can be either short or long-lived. For long-lived pores a scaling relation between the lifetime of a pore and the size of the vesicle is found. The full phase diagram of pore stability in the concentration-vesicle size plane is constructed, and the different scaling regimes are deduced analytically.

The paper is organized as follows: In Sec. II we review the previously derived equations governing the nucleation and growth of a pore in an osmotically stressed vesicle [2]. In Sec. III a numerical solution of the dynamical equations is presented. In Sec. IV the phase diagram for different dynamical regimes is derived. In Sec. V the rate of solute leak-out is determined and analytical estimates of the pore lifetime are provided. Finally, in Sec. VI the conclusions are presented.

\section{MODEL}

As was already stressed in the Introduction, the liposomal membrane allows for a free exchange of water between the exterior and interior of a vesicle. The rate of this exchange is determined by the permeability of the membrane, $P$. On the other hand, the lipidic membrane strongly inhibits the exchange of solute molecules between the inside and outside of a liposome. When a vesicle of high internal solute concentration is placed inside a solute-depleted medium, an osmotic pressure difference causes an influx of water into the vesicle. The vesicle then swells until the internal Laplace pressure is able to compensate for the osmotic pressure. The influx of water results in a buildup of membrane stress which energetically favors membrane rupture and the formation of pores. Pores are nucleated in the membrane through thermal fluctuations. Here we consider the opening of a single pore. The underlying assumption is that, once a pore is formed, stress is quickly released and the creation of a second pore becomes highly unlikely. This situation is quite different from what is encountered in electroporation. In that case the opening of a pore does not fully release the membrane stress, which is induced by the transmembrane potential, and one finds a coexistence of pores with different sizes $[3,4]$.

The single-pore assumption allows us to write simple equations governing the internal vesicle dynamics. Designating the difference between the internal and external molar concentrations of solute as $c$-and considering, for mathematical simplicity, a spherical vesicle of radius $R$ and a circular pore of radius $r$ - the mass conservation leads to

$$
4 \pi \rho R^{2} \frac{d R}{d t}=j_{w}-\pi r^{2} \rho v
$$

where $\rho$ is the density of water, $j_{w}$ the osmotic current, and $v$ is the leak-out velocity.

The osmotic current $j_{w}$ is determined by the permeability of the liposomal membrane and the difference between the target osmotic pressure,

$$
\Delta p_{o}=k_{B} T N_{A} c,
$$

and the Laplace pressure, 


$$
\Delta p_{L}=\frac{2 \sigma}{R}
$$

inside and outside the vesicle. In the above expressions $k_{B}$ is the Boltzmann constant, $T$ is temperature, $N_{A}$ is the Avogadro number, and $\sigma$ is the membrane surface tension. A simple phenomenological expression for the osmotic current of water into the vesicle is

$$
j_{w}=P\left(4 \pi R^{2}-\pi r^{2}\right)\left[c-\frac{\Delta p_{L}}{10^{3} k_{B} T N_{A}}\right],
$$

where the conversion factor $10^{3}$ accounts for the use of molar concentration of solute $c$.

If $c$ is not too large, the membrane integrity will not be compromised, and a stationary state with $j_{w}=0$ will be achieved. Under these conditions the osmotic pressure is completely compensated for by the Laplace pressure, resulting in a zero net flux of solvent. For sufficiently large internal concentration of solute, a stationary state will not be achieved before membrane ruptures. The leak-out velocity [5-8] of the internal content of a liposome is determined by the balance between the shear stress, proportional to $\eta v / r$, and the Laplace pressure inside the vesicle, $\Delta p_{L}$. For low Reynolds numbers [5],

$$
v=\frac{\Delta p_{L} r}{3 \pi \eta},
$$

where $\eta$ is the solvent viscosity.

The growth of a pore is controlled by the rate at which the membrane elastic energy is dissipated. Since the viscosity of the membrane is five orders of magnitude larger than that of water, most of the energy dissipation is confined to the membrane interior [9]:

$$
\eta_{m} l \frac{d r}{d t}=-\frac{\partial E}{\partial r}
$$

where $l$ is the membrane width and $\eta_{m}$ is the membrane viscosity. A lipid bilayer has low permeability to solute particles, in particular if they are charged, so that the internal solute concentration is modified only through the osmotic influx of solvent or the efflux of solute through an open pore, after the membrane has ruptured. The continuity equation expressing this is

$$
\frac{4 \pi}{3} R^{3} \frac{d c}{d t}=-4 \pi R^{2} c \frac{d R}{d t}-\pi r^{2} c v
$$

where we have assumed that the solute is uniformly distributed inside the vesicle. In the absence of a pore, efflux is zero, and the second term on the right-hand side of Eqs. (1) and (7) disappears.

\section{A. Membrane energy}

The membrane energy consists of two terms: the elastic term $E_{s}$, measuring the cost of increasing the membrane area beyond its equilibrium unstretched size $A_{0}$, and the pore contribution $E_{p}$, resulting from the partial exposure of the hydrophobic lipidic tales to the aqueous environment.
TABLE I. Characteristic values for the physical parameters used in the calculations.

\begin{tabular}{ccc}
\hline \hline Parameter & Value & Source \\
\hline$\gamma$ & $10^{-12} \mathrm{~J} / \mathrm{m}$ & Ref. [8] \\
$\kappa$ & $0.2 \mathrm{~J} / \mathrm{m}^{2}$ & Ref. [11] \\
$\eta_{m}$ & $100 \mathrm{~Pa} \mathrm{~s}$ & Ref. [8] \\
$\eta_{w}$ & $0.001 \mathrm{~Pa} \mathrm{~s}$ & \\
$P$ & $1.8 \times 10^{-4} \mathrm{~kg} /\left(\mathrm{m}^{2} \mathrm{~s} \mathrm{M}\right)$ & Ref. [13] \\
$l$ & $3.5 \mathrm{~nm}$ & Ref. [8] \\
\hline \hline
\end{tabular}

For large osmotic pressures, which are of interest to us, the membrane thermal undulations can be ignored and the membrane elastic energy takes a Hooke-like form

$$
E_{s}(R, r)=\frac{1}{2 A_{0}} \kappa\left(A-A_{0}\right)^{2},
$$

where $A_{0}=4 \pi R_{0}^{2}$ is the equilibrium surface area of an unstretched vesicle, $A=4 \pi R^{2}-\pi r^{2}$ is the total membrane area, and $\kappa$ is the membrane elastic modulus [10-12]. The membrane surface tension is

$$
\sigma=\frac{\partial E_{s}}{\partial A}=\kappa \frac{A-A_{0}}{A_{0}},
$$

and the pore energy is

$$
E_{p}(r)=2 \pi \gamma r
$$

where $\gamma$ is the pore line tension.

The typical values for the physical constants involved in the model are given in Table I.

\section{B. Rupture condition}

The total energy of a membrane containing a pore is $E(R, r)=E_{s}(R, r)+E_{p}(r)$. A cost of opening a pore of radius $r$ is, then,

$$
\Delta E(R, r)=E(R, r)-E(R, 0) .
$$

In Fig. 1 we plot $\Delta E(R, r)$ as a function of $r$ for various ratios of $R / R_{0}$. For $R \simeq R_{0}$, the membrane is relaxed and $r=0$ is the only minimum of $\Delta E$. For $R$ bigger than the critical radius $R_{c}$, the energy cost function develops a barrier located at

$$
r_{b}=\frac{4 \sqrt{R^{2}-R_{0}^{2}}}{\sqrt{3}} \cos \left(\frac{\varphi-2 \pi}{3}\right)
$$

and a new minimum at

$$
r_{m}=\frac{4 \sqrt{R^{2}-R_{0}^{2}}}{\sqrt{3}} \cos \left(\frac{\varphi}{3}\right),
$$

where 


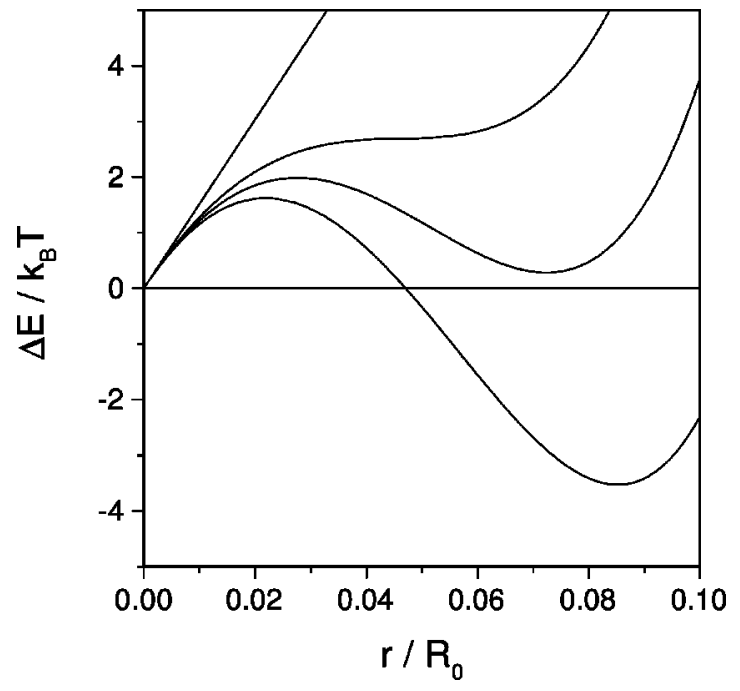

FIG. 1. Energy $\Delta E(R, r)$ necessary to open a pore of radius $r$ in a liposome with $R_{0}=100 \mathrm{~nm}$ and ratio $\gamma /\left(\kappa R_{0}\right)=5 \times 10^{-5}$. The curves are, from top down, $R / R_{0}=1.0, R / R_{0}=1.0008, R / R_{0}$ $=1.001$, and $R / R_{0}=1.0012$.

$$
\varphi\left(R, R_{0}\right)=\cos ^{-1}\left(-\frac{3 \sqrt{3}}{8} \frac{E\left(R, 2 \sqrt{R^{2}-R_{0}^{2}}\right)}{E(R, 0)}\right) .
$$

The critical vesicle size $R_{c}$ for the appearance of a new minimum is determined by the condition $\varphi=\pi$ or

$$
\frac{3 \sqrt{3}}{8} \frac{E\left(R_{c}, 2 \sqrt{R_{c}^{2}-R_{0}^{2}}\right)}{E\left(R_{c}, 0\right)}=1 .
$$

Substituting the solution of Eq. (15) into Eq. (9) the critical surface tension for appearance of the second minimum is

$$
\sigma_{c}^{(1)}=3\left(\frac{\gamma \sqrt{\kappa}}{R_{0}}\right)^{2 / 3} \text {. }
$$

From Eq. (4) we see that a minimum solute concentration

$$
c_{\min }^{(1)}=\frac{2 \sigma_{c}^{(1)}}{10^{3} k_{B} T N_{A} R_{0} \sqrt{1+\sigma_{c}^{(1)} / \kappa}}
$$

is necessary to develop the second minimum in $\Delta E$ at $r_{m}$. However, the concentration $c_{\text {min }}^{(1)}$ does not guarantee the opening of a pore. Even if $\Delta E\left(R, r_{m}\right)<0$, the energy barrier to pore nucleation can be many $k_{B} T$ high. Therefore, pores with a radius less than $r_{b}$ will quickly reseal, without having a chance to grow.

The probability of occurrence of a sufficiently large thermal fluctuation necessary to open a pore with $r>r_{b}$ is

$$
P(r) \sim e^{-\beta \Delta E\left(R, r_{b}\right)} .
$$

The waiting time for opening a pore of radius $r \geqslant r_{b}$ is, therefore, very long unless

$$
\Delta E\left(R_{p}, r_{b}\right) \simeq k_{B} T
$$

This equation, then, determines the size of a swollen vesicle,

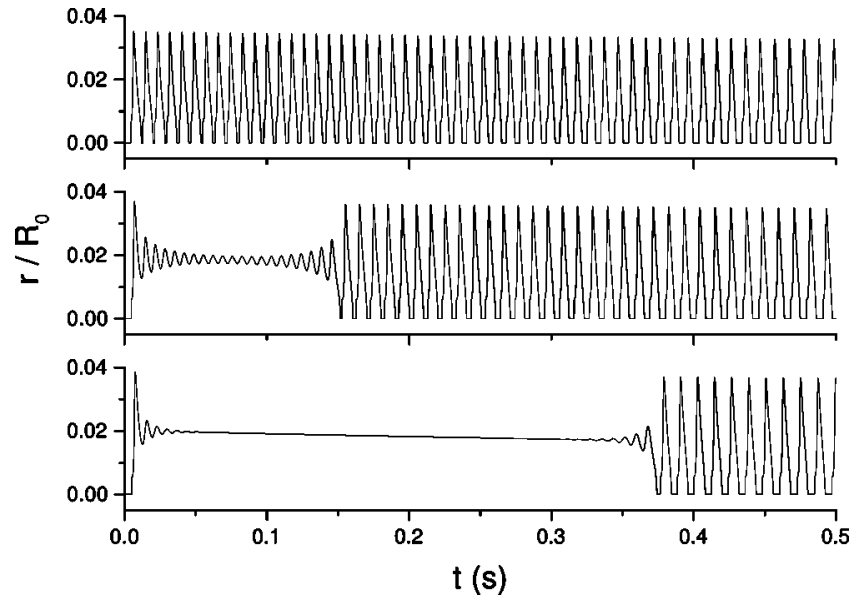

FIG. 2. Radius of a pore as a function of time for vesicles of $R_{0}=200 \mathrm{~nm}$ (topmost), $220 \mathrm{~nm}$, and $240 \mathrm{~nm}$ (bottommost), for initial concentration $c_{0}=0.5 \mathrm{M}$. Note that for a vesicle with $R_{0}$ $=200 \mathrm{~nm}$ the pores are short lived, while for larger vesicles, a longlived pore opens first.

$$
R_{p}=R_{0} \sqrt{1+\sigma_{c}^{(2)} / \kappa},
$$

which is able to nucleate a growing pore. The membrane tension of such a liposome is approximately,

$$
\sigma_{c}^{(2)} \approx \frac{\pi \gamma^{2}}{k_{B} T},
$$

and the critical pore size is $r_{b} \approx \gamma / \sigma$. The minimum concentration of solute necessary to reach this tension is

$$
c_{\text {min }}^{(2)}=\frac{2 \sigma_{c}^{(2)}}{10^{3} k_{B} T N_{A} R_{p}} .
$$

We note that for the membrane parameters given in Table $\mathrm{I}$, the membrane tension is $\sigma_{c}^{(2)} \simeq 10^{-3} \mathrm{~J} / \mathrm{m}^{2}$, which is very close to the one found to be necessary to rupture a mechanically stretched membrane [3,14].

\section{PORE DYNAMICS}

The rupture dynamics of an osmotically stressed vesicle proceeds as follows. At $t=0$ the vesicle starts swelling, its size and internal concentration controlled by Eqs. (1) and (7). As it swells, the membrane surface tension increase until $\sigma$ $=\sigma_{c}^{(1)}$ and the energy function develops a new minimum. If the barrier height is less than $k_{B} T$, a pore of size $r_{b}$ is nucleated. From this moment the dynamics of the vesicle evolution is controlled by the set of equations (1), (6), and (7). On the other hand, if $\Delta E\left(R_{c}, r_{b}\right)>k_{B} T$, the swelling continues without a pore nucleation until Eq. (19) is satisfied and a pore of radius $r_{b}$ opens. After a pore is nucleated, the internal content of the vesicle begins to leak out, decreasing the membrane tension and leading to an eventual resealment of the pore. The cycle will be repeated until the internal concentration of solute drops below $c_{\text {min }}^{(1)}$ and a steady state with $j_{w}=0$ is established. In Fig. 2 we show the pore radius as a function of time for vesicles of three different sizes and initial concentration of solute $c_{0}=0.5 \mathrm{M}$. 


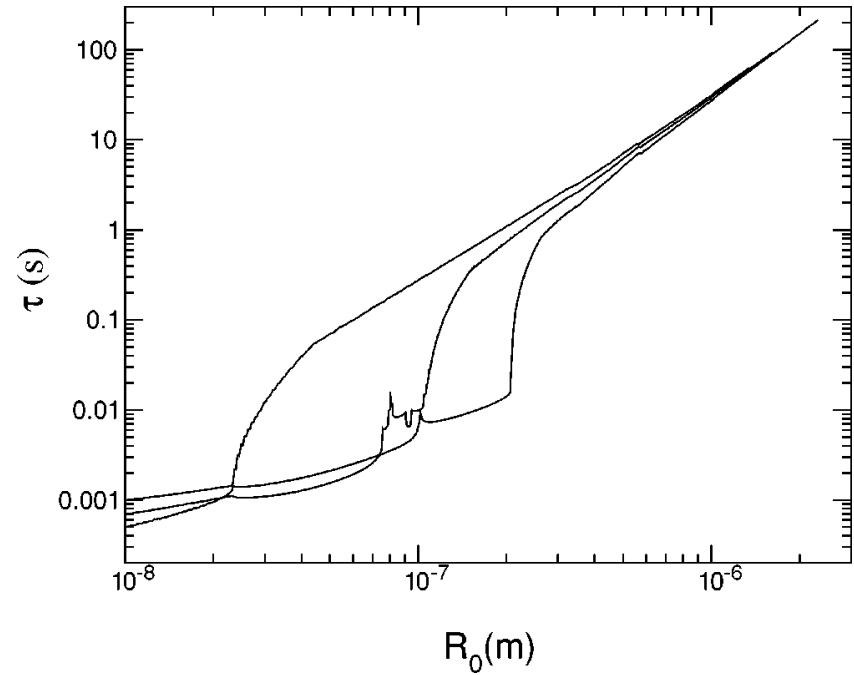

FIG. 3. Life span of the first open pore as a function of vesicle size $R_{0}$ for $c_{0}=0.5 \mathrm{M}$ (rightmost), $1.0 \mathrm{M}$ and $5.0 \mathrm{M}$ (leftmost). Note the appearance of critical vesicle size $R_{0}^{c}\left(c_{0}\right)$ (sharp change in slope of $\tau$ vs $R_{0}$ ) which sustains a long-lived pore. The peculiar spikes in the lifetime of vesicles containing a low concentration of solute are an artifact of the way the pores are nucleated. On the other hand, the existence of $R_{0}^{c}\left(c_{0}\right)$ is independent of pore nucleation protocol.

We see that small vesicles are characterized by a rapid opening and closing of pores, resulting in a periodic flickering with a characteristic time $\tau_{f} \approx 10^{-2} \mathrm{~s}$. On the other hand, larger vesicles are capable of nucleating a long-lived pore.

After the long-lived pore has closed, it is followed by a sequence of short-lived pores, with the characteristic life span $\tau_{f}$. The life span of a long-lived pore $\tau$ is show in Fig. 3. For large vesicles, the life span scales with the vesicle size as

$$
\tau \sim R_{0}^{\nu}
$$

with $\nu \approx 2.3-2.4$; see Fig. 3 .

\section{PHASE DIAGRAM}

To better understand the details of the vesicle evolution, it is convenient to separate the membrane dynamics from the concentration dynamics. Since the internal solute concentration changes very slowly compared to the $\tau_{f}$ (see Fig. 4), as a first approximation we can take it to be constant.

In this case the vesicle evolution is controlled by Eqs. (1) and (6), which can be written as

$$
\begin{aligned}
& \frac{d R}{d t}=F(R, r), \\
& \frac{d r}{d t}=G(R, r),
\end{aligned}
$$

where

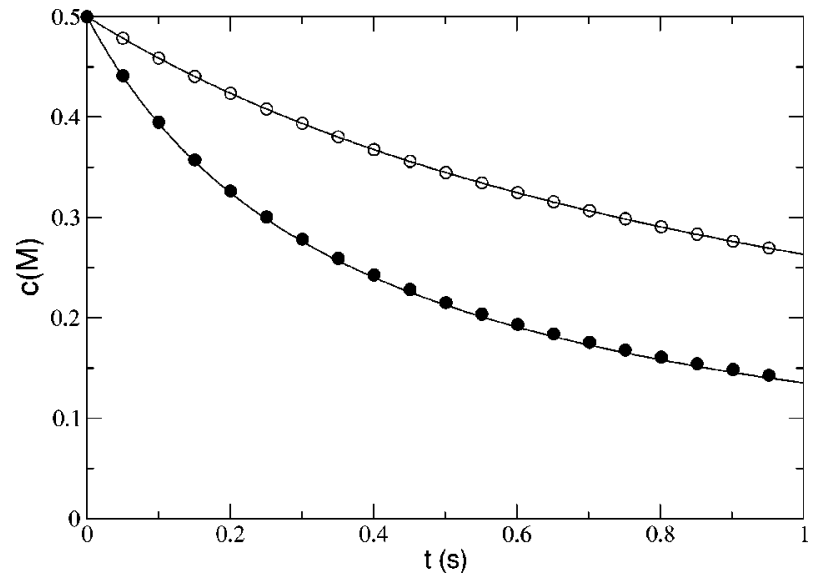

FIG. 4. Concentration decay as a function of time. The circles are the result of numerical integration of Eqs. (1), (6), and (7). The solid lines are from the analytical expression (40). Open circles are for a vesicle of $R_{0}=100 \mathrm{~nm}$ and solid circles are for a vesicle of $R_{0}=300 \mathrm{~nm}$. The initial solute concentration is $c_{0}=0.5 \mathrm{M}$.

$$
F(R, r)=\frac{1}{4 \pi \rho R}\left(j_{w}-\pi r^{2} \rho v\right)
$$

and

$$
G(R, r)=-\frac{1}{\eta_{m} \ell} \frac{\partial E}{\partial r} .
$$

The vesicle dynamics is governed by the fixed point $\left(r^{*}, R^{*}\right)$, determined from $d r / d t=0=G\left(R^{*}, r^{*}\right)=0$ and $d R / d t=0$ $=F\left(R^{*}, r^{*}\right)$. The stability of the fixed point is controlled by the eigenvalues $\lambda_{1}$ and $\lambda_{2}$ of the Jacobian matrix

$$
J=\left(\begin{array}{ll}
\frac{\partial F}{\partial R} & \frac{\partial F}{\partial r} \\
\frac{\partial G}{\partial R} & \frac{\partial G}{\partial r}
\end{array}\right) .
$$

It is important to keep in mind that the coefficients of the Jacobian matrix are real and, therefore, the eigenvalues are either real or complex conjugates, $\lambda_{1}=\bar{\lambda}_{2}$. For all the parameters that we have investigated the eigenvalues are complex conjugates, and the stability is governed by $\operatorname{Re}\left(\lambda_{1}\right)=\operatorname{Re}\left(\lambda_{2}\right)$ $\equiv \operatorname{Re}(\lambda)$. If $\operatorname{Re}(\lambda)<0$, the fixed point is stable and a stationary state with a pore of size $r^{*}$ and vesicle of size $R^{*}$ will be established. On the other hand, if $\operatorname{Re}(\lambda) \geqslant 0$, the fixed point is unstable, and the pore will eventually close; see Figs. 5 and 6. A new pore will open when the membrane tension again reaches the value $\sigma_{c}^{(2)}$. This process will repeat indefinitely with characteristic time $\tau_{f}$.

The phase boundary, in the concentration-size plane $\left(R_{0}, c\right)$, between the two dynamical regimes is determined by the condition $\operatorname{Re}(\lambda)=0$ or, equivalently,

$$
\left.\operatorname{Tr} J\right|_{R^{*}, r^{*}}=0,
$$

which reduces to 

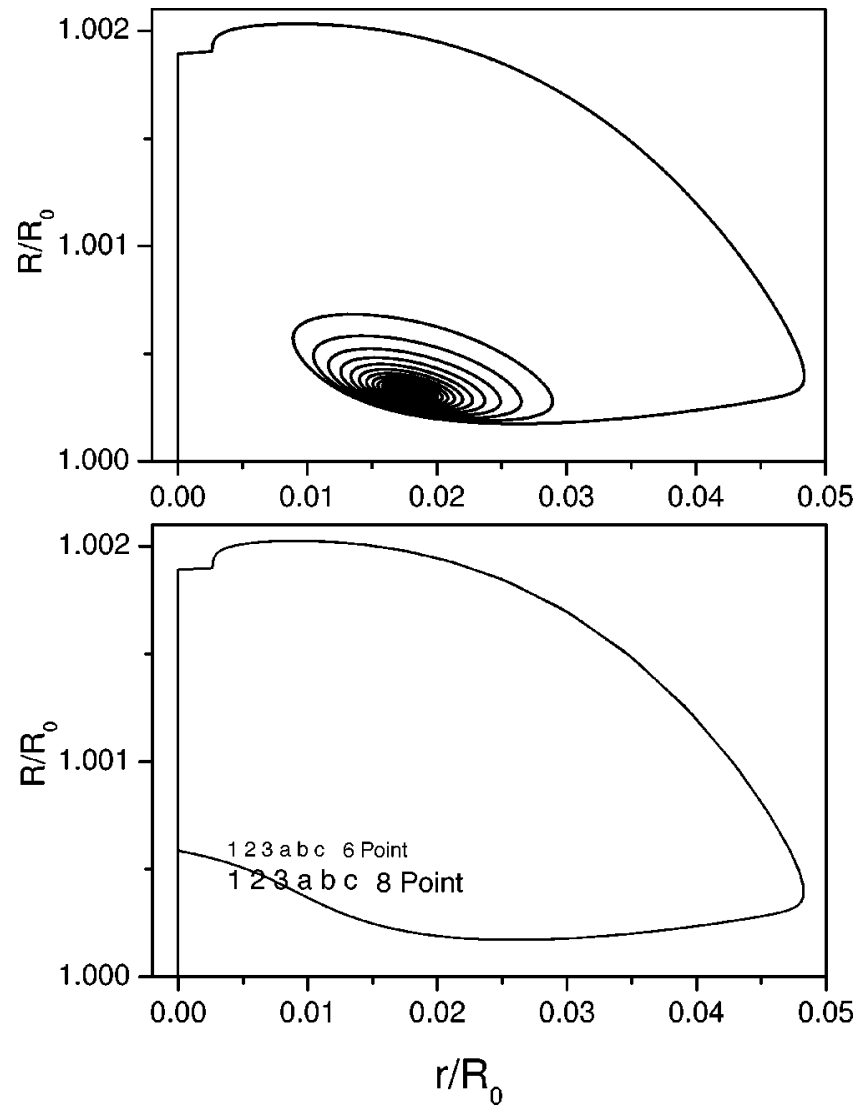

FIG. 5. Dynamics of the pore for fixed concentration and $R_{0}$ $=500 \mathrm{~nm}$. The top panel is for $c_{0}=0.19 \mathrm{M}$, and the bottom panel for $c_{0}=0.18 \mathrm{M}$.

$$
\frac{1}{4 \pi \rho R^{2}} \frac{\partial}{\partial R}\left(j_{w}-\pi r^{2} \rho v\right)-\left.\frac{1}{\eta_{m} \ell} \frac{\partial^{2} E}{\partial r^{2}}\right|_{R^{*}, r^{*}}=0 .
$$

Combining Eqs. (3) and (5), the leak-out velocity is

$$
v=\frac{2 \sigma r}{3 \pi \eta R} .
$$

Differentiating $v$ with respect to the vesicle size,

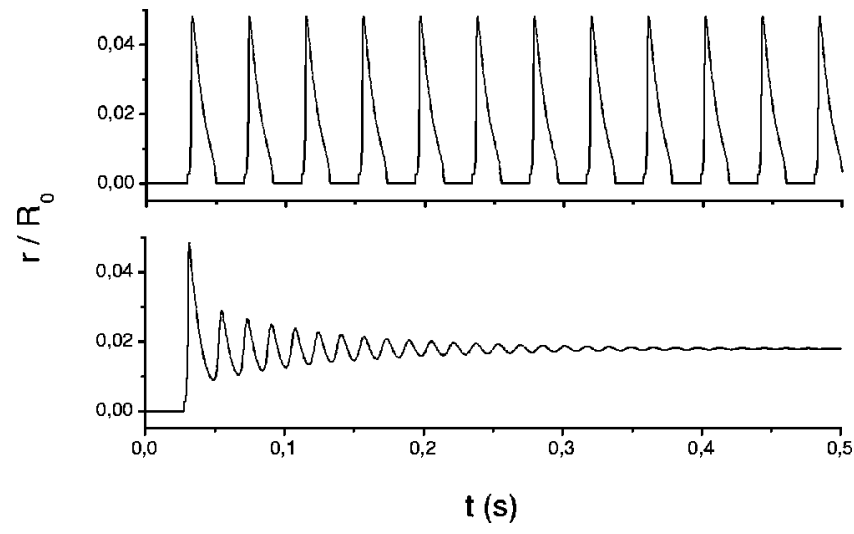

FIG. 6. Radius of a pore as a function of time for vesicles of $R_{0}=500 \mathrm{~nm}$ for a constant internal concentration of solute $c_{0}$ $=0.18 M$ (top) and $c_{0}=0.19 M$ (bottom).

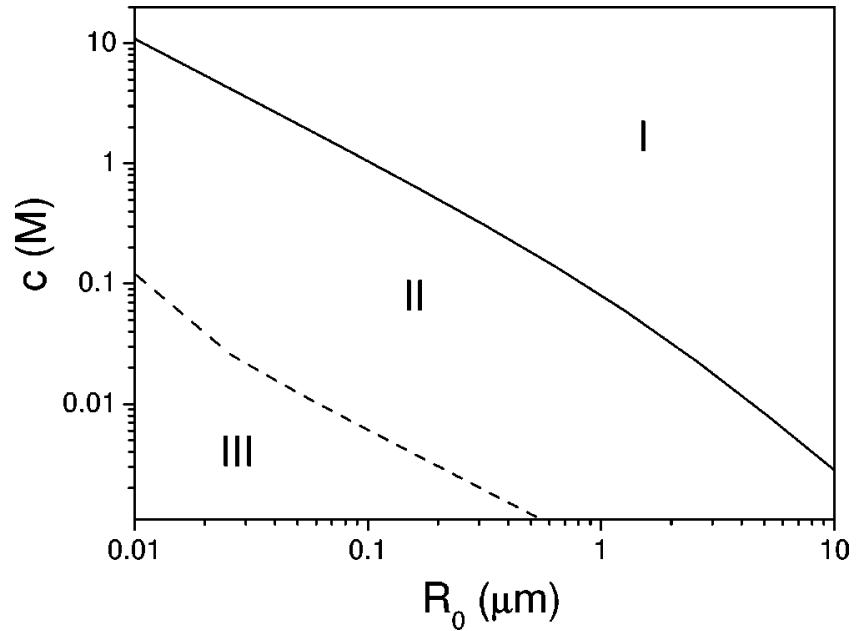

FIG. 7. The phase diagram for the constant-concentration dynamics. In region I, an open pore is stable. In region II, the size of a pore oscillates, and in region III pores do not open. The dashed line indicates the minimum solute concentration needed to open a pore. The discontinuity in its derivative is due to the fact that for small $R_{0}$ the barrier to pore nucleation is lower than $k_{B} T$.

$$
\frac{\partial v}{\partial R}=-\frac{v}{R}+\frac{16 r \kappa}{3 \eta A_{0}}
$$

The change of surface tension with the pore size is given by

$$
\frac{\partial \sigma}{\partial r}=-2 \pi r \frac{\kappa}{A_{0}}
$$

For large concentrations and $R \gg r$, the osmotic current can be approximated by

$$
j_{w} \simeq P A c,
$$

so that

$$
\frac{\partial j_{w}}{\partial R}=\frac{2}{R} j_{w}
$$

At fixed point $\left(R^{*}, r^{*}\right)$,

$$
j_{w}^{*}=\pi r^{* 2} \rho v^{*}=\frac{2 \rho \gamma^{3}}{3 \eta R_{0} \sigma^{* 2}},
$$

where we have approximated $R \simeq R_{0}$ and $r^{*}=r_{b} \simeq \gamma / \sigma^{*}$. Equation (30) then reduces to a quartic equation for $\sigma^{*}$ :

$$
\sigma^{* 4}+\frac{2 \gamma^{2}}{A_{0}}\left(\frac{\eta_{m} \ell \gamma}{\eta R_{0}^{2}}-\pi \kappa\right) \sigma^{*}-\frac{16 \pi}{3} \frac{\eta_{m} \ell \gamma^{3} \kappa}{\eta A_{0}^{2}}=0 .
$$

Combining Eqs. (34) and (36), the phase boundary separating the regime of short-lived pores (region II) from the longlived (infinite life-time) pores (region I) is

$$
c_{c}\left(R_{0}\right)=\frac{\rho \gamma^{3}}{6 \pi \eta P R_{0}^{3} \sigma^{*}}
$$

(see Fig. 7) 
For small concentrations, the surface tension does not build sufficiently high to cause the membrane rupture (region III). The phase boundary between regions II and III is denoted by a dashed curve in Fig. 7. The discontinuity in slope results from the nucleation barrier passing the threshold $\Delta E=k_{B} T$. Thus the right-hand side of the II-III phase boundary is given by $c_{\text {min }}^{(2)}\left(R_{0}\right)$, while the left-hand side $c_{\text {min }}^{(1)}\left(R_{0}\right)$.

The role of the concentration dynamics is to make the system traverse through the different regions of the phase diagram, controlling the time of permanence in each regime.

\section{DYNAMICS OF SOLUTE LEAK-OUT}

The critical size of a liposome $R_{0}^{c}\left(c_{0}\right)$, necessary for nucleating a long-lived pore, depends on the initial solute concentration. The larger is the solute concentration $c_{0}$, the smaller will be the size of a vesicle which supports a longlived pore. The long life span of these pores is the result of a "wash-out" effect in which the osmotic flux is almost completely compensated by the leak-out rate of solute through the pore. When the solute concentration inside the vesicle drops below the critical value $c_{c}\left(R_{0}\right)$, the long-lived pore closes. This value is insensitive to the initial solute concentration $c_{0}$, but depends strongly on the vesicle size $R_{0}$.

Combining Eqs. (1) and (7) we obtain

$$
\frac{4 \pi}{3} R^{3} \frac{d c}{d t}=-\frac{j_{w}}{\rho} c
$$

Approximating $j_{w} \simeq P c A$ and $R \simeq R_{0}$ leads to

$$
c(t)=c_{0}\left[\frac{3 P c_{0}}{\rho R_{0}} t+1\right]^{-1} .
$$

Equation (40) provides an almost perfect fit of the time dependence of the internal solute concentration (see Fig. 4). The life span of a long-lived pore can be approximated by the time it takes for the solute to go from the initial concentration $c_{0}$ to the critical concentration $c_{c}\left(R_{0}\right)$, below which the pore is no longer stable:

$$
\tau \simeq \frac{\rho R_{0}}{3 P c_{c}\left(R_{0}\right)}\left[1-\frac{c_{c}\left(R_{0}\right)}{c_{0}}\right] .
$$

It is possible to derivate two limits for the critical concentration $c_{c}\left(R_{0}\right)$ (see the Appendix). Writing

$$
c_{c}\left(R_{0}\right)=\frac{\rho \gamma}{6 \pi \eta P} \frac{1}{R_{0} f\left(R_{0}\right)}
$$

for vesicles of radius $R_{0} \simeq R_{1} \equiv \sqrt{\gamma \eta_{m} \ell / \pi \kappa \eta}$,

$$
f\left(R_{0}\right)=\left[\frac{1}{3^{1 / 4}} \sqrt{\frac{\kappa}{\gamma} R_{1}}+\frac{\sqrt{3}}{8 R_{0} R_{1}}\left(R_{0}^{2}-R_{1}^{2}\right)\right]^{2},
$$

and for $R_{0} \gg R_{1}$,

$$
f\left(R_{0}\right)=\left[\frac{1}{2} \frac{\kappa}{\gamma} \frac{1}{R_{0}}\left(R_{0}{ }^{2}-R_{1}{ }^{2}\right)\right]^{2 / 3},
$$

and therefore the life span of long-lived pores scales as

$$
\tau \sim \begin{cases}R_{0}^{2}, & R_{0} \simeq R_{1}, \\ R_{0}^{2+2 / 3}, & R_{0} \gg R_{1} .\end{cases}
$$

For the parameters used in this paper $R_{1}=41.8 \mathrm{~nm}$, so that Eq. (44) is consistent with the numerical findings (see Fig. 3).

The flickering time $\tau_{f}$ is approximately the time it takes for a vesicle to swell to size $R_{p}$ needed to induce a liposomal rupture. During the swelling, the internal concentration of solute changes very little, since $R_{p} \simeq R_{0}$, so that $c$ can be kept constant. Furthermore, for large initial solute concentrations, the osmotic current is $j_{w} \approx 4 \pi P R^{2} c$, and Eq. (1) is easily integrated, yielding

$$
\tau_{f}=\frac{\rho\left(R_{p}-R_{0}\right)}{P c}
$$

On the other hand,

$$
\sigma_{c}^{(2)}=\kappa \frac{R_{p}^{2}-R_{0}^{2}}{R_{0}^{2}} \approx \frac{2 \kappa}{R_{0}}\left(R_{p}-R_{0}\right) .
$$

Substituting Eq. (47) into Eq. (46) we obtain the expression for the flickering time:

$$
\tau_{f} \approx \frac{\rho R_{0} \sigma_{c}^{(2)}}{2 P_{c} \kappa},
$$

where $\sigma_{c}^{(2)}$ is given by Eq. (21).

\section{CONCLUSIONS}

We have presented a theory for the nucleation and growth of pores in osmotically stressed liposomal vesicles. The model predicts that depending on the internal solute concentration and the liposome size, pores can be either short lived-opening and closing with a characteristic time $\tau_{f}$ - or long lived, with their lifetime scaling with the size of the vesicle.

Long-lived pores have been observed in red blood cell ghosts $[15,16]$. No theory, up to date, was able to account for these long-lived pores. Holes were predicted to either grow indefinitely, which would result in ghost vesiculation, or to close completely [17]. Our model provides a dynamical mechanism for pore stabilization, consistent with the experimental observations. However, for the specific case of red blood cell ghosts the ratio of $\gamma / \kappa$ must be adjusted to obtain the pore size observed in experiments. This is not surprising since the real biological cells, unlike liposomes, have a complicated internal cytoskeleton, which strongly affects the membrane elasticity.

In aqueous solutions the phospholipid membranes acquire a net negative charge. At physiological concentrations, $154 \mathrm{mM}$ of $\mathrm{NaCl}$, the Debye length, however, is quite short, less than $1 \mathrm{~nm}$ and the electrostatic interactions are strongly screened [18]. We therefore do not expect that electrostatics will significantly modify the basic conclusions of our theory, beyond the renormalization of membrane line [17] and surface tension. However, further investigations in this direction are necessary and will be the subject of future work. 
Finally, up to now we have not taken into account a diffusive efflux of solute through an open pore. The characteristic time for effusion can be estimated as [19]

$$
\tau_{e} \approx \frac{R_{0}^{3}}{r D}
$$

where $D$ is the diffusion constant. Using $D \approx 10^{-9} \mathrm{~m}^{2} / \mathrm{s}$, appropriate for small organic molecules such as sucrose, and $r=r^{*} \approx \gamma / \sigma_{c} \approx 1 \mathrm{~nm}$, we see that for liposomes with $R_{0}$ $=200 \mathrm{~nm}$, the time for effusion is $\tau_{e} \approx 10^{-2} \mathrm{~s}$. This is comparable to the flicker time $\tau_{f}$. Therefore, for small vesicles effusion is an important mechanism for loss of solute. On the other hand, for large liposomes with $R_{0}=500 \mathrm{~nm}$ and above, effusion is only marginally relevant.

\section{ACKNOWLEDGMENTS}

This work was supported in part by the Brazilian agencies $\mathrm{CNPq}$ and FAPERGS.

\section{APPENDIX}

Here we present a derivation of the limiting form of the phase boundary, Eqs. (42)-(44), separating regions I and II of the phase diagram, Fig. 7. Writing

$$
\sigma^{*}=\frac{\gamma}{R_{0}} x
$$

Eq. (37) reduces to

$$
x^{4}+\Gamma_{1} x+\Gamma_{2}=0
$$

with

$$
\begin{gathered}
\Gamma_{1}=-\frac{1}{2} \frac{\kappa}{\gamma}\left(1+\frac{R_{1}}{R_{0}}\right)\left(R_{0}-R_{1}\right), \\
\Gamma_{2}=-\frac{1}{3}\left(\frac{\kappa}{\gamma} R_{1}\right)^{2},
\end{gathered}
$$

where

$$
R_{1}=\sqrt{\frac{\gamma \eta_{m} \ell}{\pi \kappa \eta}} .
$$

The solutions of a quartic equation like Eq. (A2) can be written as

$$
\begin{aligned}
& x_{1,2}=\frac{1}{2}\left[\sqrt{y} \pm \sqrt{-|y|-\frac{2 \Gamma_{1}}{\sqrt{y}}}\right], \\
& x_{3,4}=\frac{1}{2}\left[-\sqrt{y} \pm \sqrt{-|y|+\frac{2 \Gamma_{1}}{\sqrt{y}}}\right],
\end{aligned}
$$

where $y$ is the real root of the resolvent

$$
y^{3}-4 \Gamma_{2} y-\Gamma_{1}^{2}=0 .
$$

Since the surface tension is non-negative, the physically relevant solution for $x$ depends on the sign of $\Gamma_{1}$ and we can write

$$
x=\frac{1}{2}\left[\sqrt{\frac{2\left|\Gamma_{1}\right|}{\sqrt{y}}-|y|}-\operatorname{sgn}\left(\Gamma_{1}\right) \sqrt{y}\right] .
$$

For an equation of the form $y^{3}+a y+b=0$ there is a single real root if

$$
Q=\left(\frac{b}{2}\right)^{2}+\left(\frac{a}{3}\right)^{3}>0
$$

and this real root is

$$
y=\left(-\frac{b}{2}+\sqrt{Q}\right)^{1 / 3}+\left(-\frac{b}{2}-\sqrt{Q}\right)^{1 / 3} .
$$

This is precisely our case since $\Gamma_{2}<0$ and, therefore, $Q>0$. It is convenient to rewrite Eq. (A6) as

$$
y=\left(\frac{\Gamma_{1}^{2}}{2}\right)^{1 / 3} \psi(z)
$$

where

$$
z=-\left(\frac{\Gamma_{2}}{3}\right)^{3}\left(\frac{4}{\Gamma_{1}}\right)^{4}
$$

and

$$
\psi(z)=(\sqrt{1+z}+1)^{1 / 3}-(\sqrt{1+z}-1)^{1 / 3} .
$$

The $\psi$ function has the asymptotic behaviors

$$
\psi(z)= \begin{cases}\frac{2}{3} z^{-1 / 3}-\frac{8}{81} z^{-4 / 3}, & z \gg 1, \\ 2^{1 / 3}-(z / 2)^{1 / 3}, & z \ll 1 .\end{cases}
$$

Putting everything together we have

$$
y= \begin{cases}\frac{\Gamma_{1}^{2}}{4 \Gamma_{2}}, & z \gg 1, \\ \Gamma_{1}^{2 / 3}, & z \ll 1 .\end{cases}
$$

In the first case we obtain

$$
x \simeq\left(-\Gamma_{2}\right)^{1 / 4}-\frac{\Gamma_{1}}{4 \sqrt{-\Gamma_{2}}}
$$

and in the second case

$$
x \simeq\left|\Gamma_{1}\right|^{1 / 3} .
$$

Using the expressions for $\Gamma_{1}$ and $\Gamma_{2}$, we obtain Eqs. (42)-(44). 
[1] C. Taupin, M. Dvolaitzky, and C. Sauterey, Biochemistry 14, 4771 (1975).

[2] Y. Levin and M. A. Idiart, Physica A 331, 571 (2004).

[3] J. C. Weaver and Y. A. Chizmadzhev, Bioelectrochem. Bioenerg. 41, 135 (1996).

[4] V. F. Pastuchenko, Y. A. Chizmadzhev, and V. B. Arakelyan, Bioelectrochem. Bioenerg. 6, 53 (1979).

[5] J. Happel and H. Brenner, Low Reynold Number Hydrodynamics (Martinus Nijhoff, Dordrecht, 1986).

[6] D. V. Zhelev and D. Needham, Biochim. Biophys. Acta 1147, 89 (1993).

[7] O. Sandre, L. Moreaux, and F. Brochard-Wyart, Proc. Natl. Acad. Sci. U.S.A. 96, 10591 (1999).

[8] F. Brochard-Wyart, P. G. de Gennes, and O. Sandre, Physica A 278, 32 (2000).

[9] G. Debregeas, P. Martin, and F. Brochard-Wyart, Phys. Rev.
Lett. 75, 3886 (1995).

[10] W. Helfrich and R. Servus, Nuovo Cimento Soc. Ital. Fis., D 3, 1 (1984).

[11] E. Evans and W. Rawicz, Phys. Rev. Lett. 64, 2094 (1990).

[12] P. Sens and H. Isambert, Phys. Rev. Lett. 88, 128102 (2001).

[13] E. Sackmann, in Biophysics, edited by W. Hoppe, W. Lohmann, H. Markl, and H. Ziegler (Springer-Verlag, Berlin, 1983).

[14] H. T. Tien, Bilayer Lipid Membranes (Marcel Dekker, New York, 1974).

[15] T. Steck et al., Science 168, 255 (1970).

[16] T. Steck and J. Kant, Methods Enzymol. 31, 172 (1974).

[17] M. D. Betterton and M. P. Brenner, Phys. Rev. Lett. 82, 1598 (1999).

[18] Y. Levin, Rep. Prog. Phys. 65, 1577 (2002).

[19] Y. Levin, M. A. Idiart, and J. J. Arenzon (unpublished). 